\title{
Impaired riboflavin transport due to missense mutations in SLC52A2 causes Brown-Vialetto-Van Laere syndrome
}

\author{
Tobias B. Haack • Christine Makowski • Yoshiaki Yao • \\ Elisabeth Graf • Maja Hempel • Thomas Wieland • Ulrike Tauer • \\ Uwe Ahting • Johannes A. Mayr • Peter Freisinger • \\ Hiroki Yoshimatsu • Ken Inui • Tim M. Strom • \\ Thomas Meitinger • Atsushi Yonezawa $\cdot$ Holger Prokisch
}

Received: 7 May 2012 /Revised: 30 May 2012 / Accepted: 26 June 2012

(C) SSIEM and Springer 2012

\begin{abstract}
Brown-Vialetto-Van Laere syndrome (BVVLS [MIM 211530]) is a rare neurological disorder characterized by infancy onset sensorineural deafness and ponto-bulbar palsy. Mutations in SLC52A3 (formerly C20orf54), coding for riboflavin transporter 2 (hRFT2), have been identified as the molecular genetic correlate in several individuals with BVVLS. Exome sequencing of just one single case revealed that compound heterozygosity for two pathogenic mutations in the SLC52A2 gene coding for riboflavin transporter 3 (hRFT3), another member of the riboflavin transporter family, is also associated with BVVLS. Overexpression studies confirmed that the gene products of both mutant alleles have reduced riboflavin transport activities. While mutations in SLC52A3 cause decreased plasma riboflavin levels, concordant
\end{abstract}

Communicated by: K. Michael Gibson

Competing interest: None declared.

Tobias B. Haack, Christine Makowski and Yoshiaki Yao contributed equally to this work

T. B. Haack • M. Hempel • T. M. Strom • T. Meitinger •

H. Prokisch $(\bowtie)$

Institute of Human Genetics, Technische Universität München,

Trogerstrasse 22, München 81675, Germany

e-mail: prokisch@helmholtz-muenchen.de

T. B. Haack • E. Graf ' T. Wieland · T. M. Strom • T. Meitinger

H. Prokisch

Institute of Human Genetics, Helmholtz Zentrum München,

Neuherberg 85764, Germany

C. Makowski $\cdot$ U. Tauer

Department of Pediatrics, Technische Universität München,

Munich 80804, Germany

Y. Yao $\cdot$ H. Yoshimatsu $\cdot$ K. Inui $\cdot$ A. Yonezawa

Department of Pharmacy, Kyoto University Hospital,

Kyoto 606-8507, Japan with a role of $S L C 52 A 3$ in riboflavin uptake from food, the SLC52A2-mutant individual had normal plasma riboflavin concentrations, a finding in line with a postulated function of $S L C 52 A 2$ in riboflavin uptake from blood into target cells. Our results contribute to the understanding of human riboflavin metabolism and underscore its role in the pathogenesis of BVVLS, thereby providing a rational basis for a high-dose riboflavin treatment.

\section{Introduction}

Since the first report by Brown in 1894 (Brown 1894) about 60 individuals suffering from BVVLS have been described

\section{U. Ahting}

Department of Clinical Chemistry, Städtisches Klinikum

München,

Munich 80804, Germany

\section{J. A. Mayr}

Department of Paediatrics, Paracelsus Medical University

Salzburg,

Salzburg 5020, Austria

\section{P. Freisinger}

Department of Pediatrics, Klinikum Reutlingen,

Reutlingen 72764, Germany

K. Inui

Kyoto Pharmaceutical University,

Yamashina-ku, Kyoto 607-8414, Japan 
(Sathasivam 2008). Age of onset varies from infancy to the third decade. Progressive and severe sensorineural deafness is the most frequently observed presenting symptom. During the invariably progressive course of the disease involvement of the lower cranial nerves is observed in most affected individuals and upper and lower motor neuron limb signs are common neurological findings. Additional features include but are not limited to cerebellar ataxia, sensory neuropathy, optic atrophy, retinitis pigmentosa, mental retardation, and psychiatric abnormalities. Diaphragmatic paralysis causing respiratory compromise is a major cause of death (Sathasivam 2008). The heterogenous clinical spectrum overlaps with Fazio-Londe syndrome, Madras motor neuron disease, Boltshauser syndrome, amyotrophic lateral sclerosis, and Nathalie syndrome.

Mutations in SLC52A3 (GenBank accession no. NM 033409), coding for human riboflavin transporter 2 , have been recently identified as the molecular genetic correlate in a total of ten BVVLS index cases (Bosch et al 2011; Green et al 2010; Anand et al 2012). These results are especially important as they provide a mechanistic rational for a high-dose riboflavin supplementation, which was indeed effective in some individuals in improving the biochemical and clinical condition (Bosch et al 2011; Anand et al 2012).

\section{Clinical data}

Here, we report on a girl with a clinical presentation consistent with BVVLS. She was born at 36 weeks of gestation to healthy, unrelated parents. An older sister is healthy. Her psychomotor development was normal until the age of 3 years when her parents first noticed impaired hearing and walking, clumsiness, and vertical eye movements. At 3 years and 2 months of age she was admitted to hospital with acute onset truncal ataxia and vertical nystagmus. Ophthalmologic examination showed a beginning optic atrophy and impaired visual acuity. Over the next 6 months behavioral abnormalities including aggressive and auto-aggressive behavior developed, her gait became wide based and frequent falls occurred. The vertical nystagmus changed to chaotic eye movements and progressive hearing loss necessitated the usage of sign language. There was no muscle weakness and nerve conduction velocity $(\mathrm{NCV})$ of the peroneal nerve was normal $(59 \mathrm{~m} / \mathrm{s}$, amplitude $3.9 \mathrm{mV})$. However, her deep tendon reflexes in the lower extremities were absent and NCV of the sural nerve was undetectable, indicating a sensory neuropathy. Extensive laboratory testing was noncontributory and analysis of mitochondrial respiratory chain enzymes showed normal activities of all complexes in muscle.
Unsteadiness gradually worsened and she was confined to a wheelchair from the age of 5 years. Ophthalmologic examination revealed a marked atrophy of the optic nerve. A nervus suralis biopsy showed an axonal neuropathy without signs of demyelination. Audiologic testing showed absent acustic evoked potentials as well as absent potentials in brainstem evoked response audiometry indicating retrocochlear deafness. Together with progressive hearing loss her active speech production declined. Neurological examination at the age of 5.5 years showed a right-sided palsy of the facial nerve and tongue wasting with fasciculations.

\section{Methods and results}

We performed exome sequencing using the Agilent Human All Exon $50 \mathrm{Mb}$ kit for targeted enrichment and subsequent sequencing on the HiSeq2000 (Illumina) to identify the disease causing mutations. Read alignment and variant calling were performed as described previously (Mayr et al 2012). We produced a total of $12.5 \mathrm{~Gb}$ of mappable sequences and $92.8 \%$ of the target was covered $>20 x$. A filter for genes carrying compound heterozygous and homozygous variants absent from 1064 control exomes identified five genes: TMCO4, OPN4, ABLIM1, FAM187B, and SLC52A2 (Table 1).

Given the role of impaired riboflavin transport in the pathogenesis of BVVLS, one gene, SLC52A2 (GenBank accession no. NM_024531), encoding human riboflavin transporter 3 (hRFT3), clearly stood out. Both mutations, c.368T $>$ C, p.Leu123Pro and c.1016T $>$ C, p.Leu339Pro, alter amino acids evolutionarily conserved from human to Danio rerio (Fig. 1). While the c.368T $>$ C, p.Leu123Pro mutation was absent from public single nucleotide polymorphism databases, the c.1016T $>$ C, p.Leu339Pro variant (rs148234606) was found 3 times in the heterozygous state in 5375 exomes listed in the Washington Exome Variant Server. Sanger sequencing confirmed the compound heterozygous state of the mutations in the affected individual with the mother being a heterozygous carrier of the c.1016T $>C$, p.Leu339Pro and the father of the c.368T $>$ C, p.Leu123Pro mutation.

Table 1 Variants identified in individual \#54994 by exome sequencing

\begin{tabular}{ll}
\hline Synonymous & 11,199 \\
NSV & 10,486 \\
NSV absent from 1046 controls & 258 \\
$\geq 2$ NSV/gene & 5 (TMCO4, OPN4, ABLIM1, \\
& FAM187B, SLC52A2) \\
\hline
\end{tabular}

$\mathrm{NSV}=$ missense, nonsense, stop loss, splice site disruption, insertions, deletions 


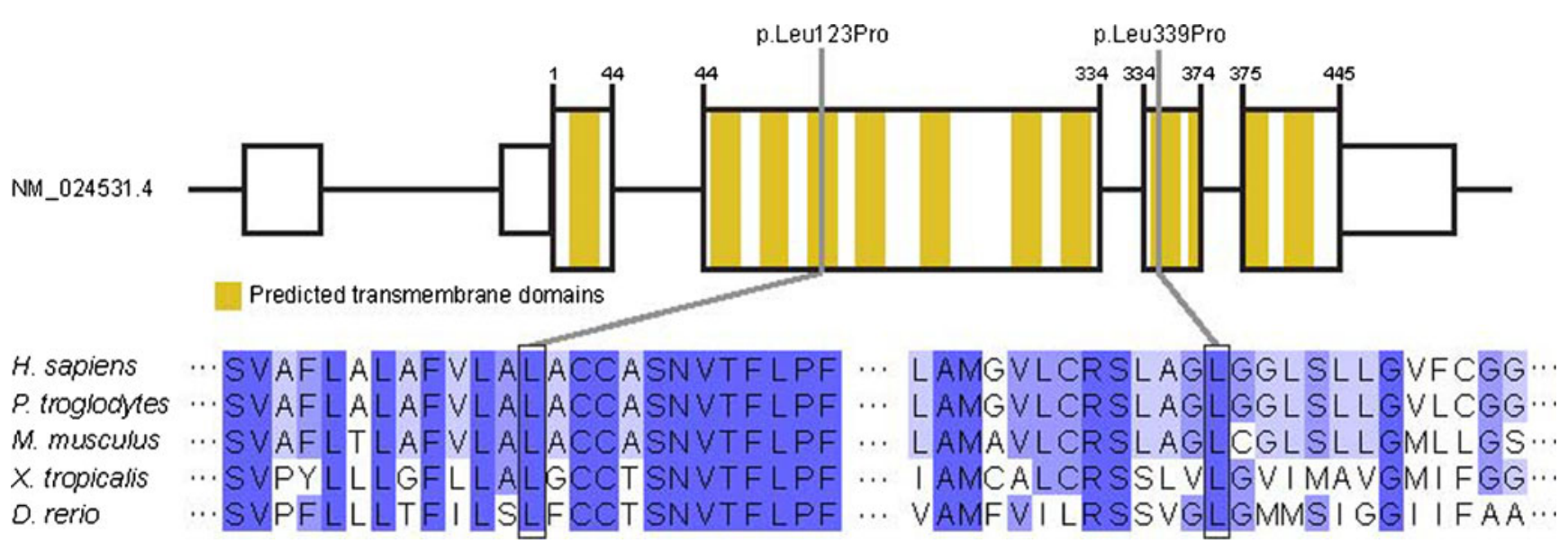

Fig. 1 Structure of SLC52A2 and localization and conservation of affected amino acid residues of identified mutations. Dark blue indicates fully conserved amino acid residues, light blue marks amino

In order to test the pathogenic impact of the mutations, we next determined the in vitro function of the two mutated hRFT3 proteins as described previously (Ho et al 2010). In brief, the human SLC52A2 wildtype $\left({ }^{\mathrm{wt}}\right)$ cDNA was subcloned into pFLAG-CMV-6a expression vector (SigmaAldrich, St. Louis, MO). Both mutations, c.368T $>$ C and c.1016T $>$ C, were introduced by site-directed mutagenesis with KOD-Plus-Mutagenesis Kit (TOYOBO, Osaka, Japan) using following primer sets, forward primer 5'- CGGCATGCTGTGCCTCGAATGTCACT-3', reverse primer 5'-GTGCCAGCACAAAGGCCAGTGCTAA-3' for $368 \mathrm{~T}>\mathrm{C}$, and forward primer $5^{\prime}-$ CGGGCAGCCTCTCTCTGCTGGGCGTG-3', reverse primer 5'-GCCCTGCCAAGGACCTGCACAGCAC-3' for 1016T $>C$. The mutations were confirmed by direct sequencing. HEK 293 cells were transfected with an empty vector and vectors containing $S L C 52 A 2^{\mathrm{wt}}, S L C 52 A 2^{368 \mathrm{~T}>\mathrm{C}}$, and $S L C 52 A 2^{1016 \mathrm{~T}>\mathrm{C}}$ using Lipofectamine 2000 (Life Technologies, Carlsbad, CA). Forty-eight hours after the transfection, the cells were used for the functional experiments. Cells were incubated with $10 \mathrm{nM}\left[{ }^{3} \mathrm{H}\right]$ riboflavin (Moravek Biochemicals, Inc., Brea, CA) for 1 min and, after washing and solubilization, radioactivity was measured using liquid scintillation counting.

Quantification of the cellular riboflavin uptake showed that both mutations cause a significant decrease in the hRFT3 transporter activity, compared to wt transfectants, thereby supporting pathogenicity (Fig. 2a). To confirm the transfection efficiency of these cells, reverse transcriptase-PCR (RT-PCR) analysis was carried out using the following primer sets, forward primer 5'-GTGGCACCATGTGGCCCCAG-3', reverse primer 5'-CAACAGCAGCAGAAGACCCT-3'. The detailed methods were previously described (Yonezawa et al 2008). The RNA expressions of $S L C 52 A 2^{368 \mathrm{~T}>\mathrm{C}}$ and $S L C 52 A 2^{1016 \mathrm{~T}>\mathrm{C}}$ expressing cell were comparable to that of $S L C 52 A 2^{\mathrm{wt}}$ - acids conserved in $60-80 \%$ of species, white indicates residues conserved in $<60 \%$ of species

expressing cell (Fig. 2b). Native SLC52A2 was only slightly observed in the cells transfected with empty vector.

Recent studies have shown that the metabolic profile of BVVLS individuals mimics a mild form of multiple acyl-

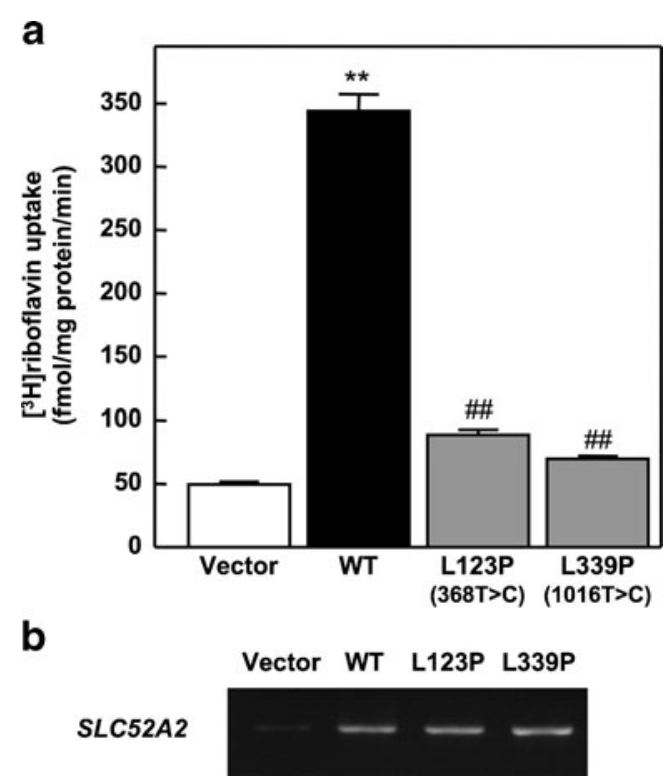

Fig. 2 In vitro activity of mutant hRFT3. a Uptake of $\left[{ }^{3} \mathrm{H}\right]$ riboflavin by HEK293 cells transfected with empty vector, $S L C 52 A 2^{\mathrm{wt}}$ (WT), SLC52A2 $2^{368 \mathrm{~T}>\mathrm{C}}$ (L123P), and SLC52A2 $2^{1016 \mathrm{~T}>\mathrm{C}}$ (L339P). The cells were incubated in buffer (pH 7.4) containing $10 \mathrm{nM}\left[{ }^{3} \mathrm{H}\right]$ riboflavin for $1 \mathrm{~min}$ at $37^{\circ} \mathrm{C}$. Each bar represents the mean \pm SEM, $n=3$. Data were analyzed by Dunnett's two-tailed test after one-way ANOVA. ** Different from vector-transfected cells, $P<0.01$. \#\# Different from $S L C 52 A 2^{\text {wt }}$-transfected cells, $P<0.01$. b RNA expression of $S L C 52 A 2$ in HEK293 cells transfected with empty vector, $S L C 52 A 2^{\mathrm{wt}}$ (WT), $S L C 52 A 2^{368 \mathrm{~T}>\mathrm{C}}(\mathrm{L} 123 \mathrm{P})$, and SLC52A2 $2^{1016 \mathrm{~T}>\mathrm{C}}$ (L339P). RT-PCR analysis was carried out using specific primer sets. Products of $360 \mathrm{bp}$ that corresponded to the SLC52A2 gene were separated by $1.5 \%$ agarose gel electrophoresis and visualized by ethidium bromide staining 
Table 2 Plasma acylcarnitines $(\mu \mathrm{mol} / \mathrm{L})$ in individual \#54994 before and after riboflavin treatment compared to reported individuals (Pat 1-3) with SLC52A3 mutations (Bosch et al 2011)
Bold values are outside normal range given in brackets. $w$ weeks, $n . d$. not determined

\begin{tabular}{|c|c|c|c|c|c|c|c|}
\hline \multirow[t]{2}{*}{ Treatment } & \multicolumn{2}{|c|}{ \#54994 } & \multirow[t]{2}{*}{ Normal range } & \multirow{2}{*}{$\begin{array}{l}\text { Pat } 1 \\
\text { No }\end{array}$} & \multirow{2}{*}{$\begin{array}{l}\text { Pat } 2 \\
\text { No }\end{array}$} & \multirow{2}{*}{$\begin{array}{l}\text { Pat } 3 \\
\text { No }\end{array}$} & \multirow[t]{2}{*}{ Normal range } \\
\hline & No & After $4 \mathrm{w}$ & & & & & \\
\hline $\mathrm{C} 0$ & n.d. & n.d. & & $18.3 \downarrow$ & 35.1 & 37.6 & $(22.0-55.0)$ \\
\hline $\mathrm{C} 2$ & 8.39 & 6.50 & $(2.94-39.78)$ & $3.2 \downarrow$ & 4.0 & $0.74 \downarrow$ & $(3.4-13.0)$ \\
\hline $\mathrm{C} 3$ & 0.38 & 0.30 & $(0.12-1.50)$ & n.d. & n.d. & n.d. & \\
\hline $\mathrm{C} 4$ & 0.19 & 0.10 & $(0.06-0.89)$ & $0.65 \uparrow$ & 2.97 & 0.43 & $(0.07-0.58)$ \\
\hline $\mathrm{C} 5$ & 0.16 & 0.07 & $(0.03-0.49)$ & $0.29 \uparrow$ & $1.99 \uparrow$ & $0.91 \uparrow$ & $(0.04-0.22)$ \\
\hline C6 & 0.07 & 0.04 & $(0.01-0.31)$ & $0.27 \uparrow$ & $0.87 \uparrow$ & 0.02 & $(0.01-0.12)$ \\
\hline $\mathrm{C} 8$ & 0.39 & 0.14 & $(0.05-0.94)$ & 0.18 & $0.70 \uparrow$ & $\mathbf{0 . 5 3} \uparrow$ & $(0.04-0.22)$ \\
\hline $\mathrm{C} 10$ & 0.35 & 0.13 & $(0.05-0.67)$ & 0.25 & 0.86 & 0.77 & $(0.04-0.30)$ \\
\hline C10:1 & $0.35 \uparrow$ & 0.09 & $(0.02-0.28)$ & n.d. & n.d. & n.d. & \\
\hline C10:2 & $1.70 \uparrow$ & 0.56 & $(0.03-1.15)$ & n.d. & n.d. & n.d. & \\
\hline $\mathrm{C} 12$ & 0.08 & 0.02 & $(0.01-0.23)$ & $0.15 \uparrow$ & $0.44 \uparrow$ & $0.64 \uparrow$ & $(0.04-0.12)$ \\
\hline $\mathrm{C} 14$ & 0.04 & 0.02 & $(0.01-0.23)$ & $0.14 \uparrow$ & $0.23 \uparrow$ & $0.46 \uparrow$ & $(<0.08)$ \\
\hline C14:1 & $2.47 \uparrow$ & 0.69 & $(0.06-1.74)$ & n.d. & n.d. & n.d. & \\
\hline $\mathrm{C} 14: 2$ & $0.76 \uparrow$ & 0.41 & $(0.02-0.67)$ & n.d. & n.d. & n.d. & \\
\hline $\mathrm{C} 14-\mathrm{OH}$ & $0.24 \uparrow$ & 0.05 & $(<0.24)$ & n.d. & n.d. & n.d. & \\
\hline $\mathrm{C} 16$ & 0.13 & 0.10 & $(0.06-0.73)$ & 0.19 & 0.19 & $0.32 \uparrow$ & $(0.06-0.24)$ \\
\hline C16:1 & 0.45 & 0.32 & $(0.04-0.79)$ & n.d. & n.d. & n.d. & \\
\hline $\mathrm{C} 16-\mathrm{OH}$ & $0.19 \uparrow$ & 0.04 & $(<0.17)$ & n.d. & n.d. & n.d. & \\
\hline C16:1-OH & $0.11 \uparrow$ & $0.14 \uparrow$ & $(<0.11)$ & n.d. & n.d. & n.d. & \\
\hline C18:1 & 1.13 & 0.83 & $(0.11-2.27)$ & 0.16 & 0.13 & $0.42 \uparrow$ & $(0.06-0.28)$ \\
\hline
\end{tabular}

CoA dehydrogenation defects (MADD) (Bosch et al 2011). Analysis of plasma acylcarnitine levels in this patient demonstrated moderately elevated levels of several acylcarnitine and hydroxy-acylcarnitine species (Table 2). We did not find any changes in urine organic acids and plasma riboflavin, flavin mononucleotide (FMN) or flavin adenine dinucleotide (FAD) concentrations (Table 3). Based on our results and the positive response reported in several BVVLS individuals (Anand et al 2012; Bosch et al 2011), we started an oral riboflavin supplementation $(10 \mathrm{mg} / \mathrm{kg}$ body weight/day). Clinical and laboratory follow up examination after 4 weeks showed that her fine motor skills and assisted gait had improved, and levels of most acylcarnitine species were now within normal range (Table 2).

\section{Discussion}

Riboflavin, also known as vitamin $\mathrm{B}_{2}$, is a water-soluble aromatic substance and has to be absorbed from the food. In humans, at least two riboflavin transporters strongly expressed in small intestine epithelial cells, hRFT1 and hRFT2 encoded by SLC52A1 (GenBank accession no. NM_017986) and SLC52A3, respectively, cooperatively function in riboflavin uptake and subsequent distribution into the blood (Yao et al 2010). Uptake into the target cells from the blood might occur via passive diffusion but more likely depends on specific transport systems (Foraker et al 2003). Inside the cells, riboflavin is converted into its bioactive coenzymes FMN and FAD. The latter functions as an electron acceptor for numerous dehydrogenases involved,

Table 3 Plasma riboflavin, FAD, and FMN levels (nmol/L) in individual \#54994 before and after riboflavin treatment compared to reported individuals (Pat 1-3) with SLC52A3 mutations (Bosch et al 2011)

\begin{tabular}{|c|c|c|c|c|c|c|c|}
\hline \multirow[t]{2}{*}{ Treatment } & \multicolumn{2}{|c|}{ \#54994 } & \multirow[t]{2}{*}{ Normal range } & \multirow{2}{*}{$\begin{array}{l}\text { Pat } 1 \\
\text { No }\end{array}$} & \multirow{2}{*}{$\begin{array}{l}\text { Pat } 2 \\
\text { No }\end{array}$} & \multirow{2}{*}{$\begin{array}{l}\text { Pat } 3 \\
\text { No }\end{array}$} & \multirow[t]{2}{*}{ Normal range } \\
\hline & No & After $4 \mathrm{w}$ & & & & & \\
\hline Riboflavin & 7.4 & $132.7 \uparrow$ & & $1.4 \downarrow$ & $<1 \downarrow$ & 7.6 & $(3.9-49)$ \\
\hline FMN & 10.1 & $17.5 \uparrow$ & & $1.7 \downarrow$ & $<1 \downarrow$ & $<1 \downarrow$ & $(2.8-11.0)$ \\
\hline FAD & 325.6 & $399.7 \uparrow$ & $(229.1-375.5)$ & $31 \downarrow$ & $30 \downarrow$ & $24.5 \downarrow$ & $(46-114)$ \\
\hline
\end{tabular}

Bold values are outside normal range given in brackets. $w$, weeks 
Fig. 3 Potential role of hRFT3 in riboflavin metabolism. Riboflavin is taken up from nutrition and passed through into the blood by intestinal epithelial cells. Proposed transporters involved are hRFT1 and hRFT2. Uptake into the target cells might occur to a small amount via passive diffusion but likely essentially relies on specific transport systems. hRFT3 is highly expressed in the brain, concordant with an important role in brain riboflavin homeostasis. In the cells, riboflavin is converted into the dehydrogenation reaction coenzymes FMN and FAD. Lack of FAD results in impaired acyl-CoA dehydrogenation leading to an accumulation acylcarnitines as found in ETFDH or ETF mutant individuals presenting with MADD. Superfluous riboflavin undergoes renal excretion. CACT, carnitine-acylcarnitine tranlsocase; CPT2, carnitine O-palmitoyl transferase 2; ETF, electron transfer flavoprotein; ETFDH, ETF-dehydrogenase; MIM, mitochondrial inner membrane

e.g., in mitochondrial fatty acid oxidation and branched chain amino acid catabolism. In rats, severe riboflavin deficiency mimics the metabolic signature of a multiple acylcoA dehydrogenation defect (MADD) (Goodman 1981). Both, decreased levels of riboflavin and its metabolites and biochemical findings suggestive for mild MADD have recently been reported in hRFT2 mutant BVVLS individuals (Bosch et al 2011). Plasma acylcarnitines and urine organic acids levels were corrected to normal values upon oral riboflavin supplementation and clinical features such as muscle weakness improved within weeks (Anand et al 2012; Bosch et al 2011). In addition to these patients, another study had investigated a patient with a complex neonatal neurological disorder and a biochemical profile suggestive for MADD (Ho et al 2010). Sequence analysis of SLC52A1 revealed that the mother carried a heterozygous deletion of exons 2 and 3. During pregnancy, this defect led to a haploinsufficiency in riboflavin transport in the mother with subsequent riboflavin deprivation of the infant. Clinical signs and biochemical abnormalities completely resolved after riboflavin treatment.

Here, we report causal mutations in a third member of the riboflavin transporter family. SLC52A2 is located on chromosome 8 and encodes a 445 amino acid protein containing 11 predicted membrane-spanning domains (Fig. 1). It has been initially reported to act as a receptor for porcine endogenous retrovirus subgroup A. Based on its amino acid sequence similarity with hRFT1 (86.7\%) and hRFT2 $(44.1 \%)$ a physiological function in riboflavin transport has been postulated and was subsequently confirmed by $\left[{ }^{3} \mathrm{H}\right]$ riboflavin uptake experiments. The protein was therefore proposed to be renamed hRFT3. Expression analysis had shown that $S L C 52 A 2$ is highly expressed in human brain and salivary gland, whereas the other two members of the riboflavin transporter family are strongly expressed in the small intestine. These findings suggested a role of hRFT3 in brain riboflavin homeostasis (Yao et al 2010). While mutant hRFT2 results in decreased plasma levels of riboflavin and its coenzyme forms FMN and FAD (Bosch et al 2011), riboflavin and its derivates were within normal range in the presented hRFT3-mutant individual. However, slightly

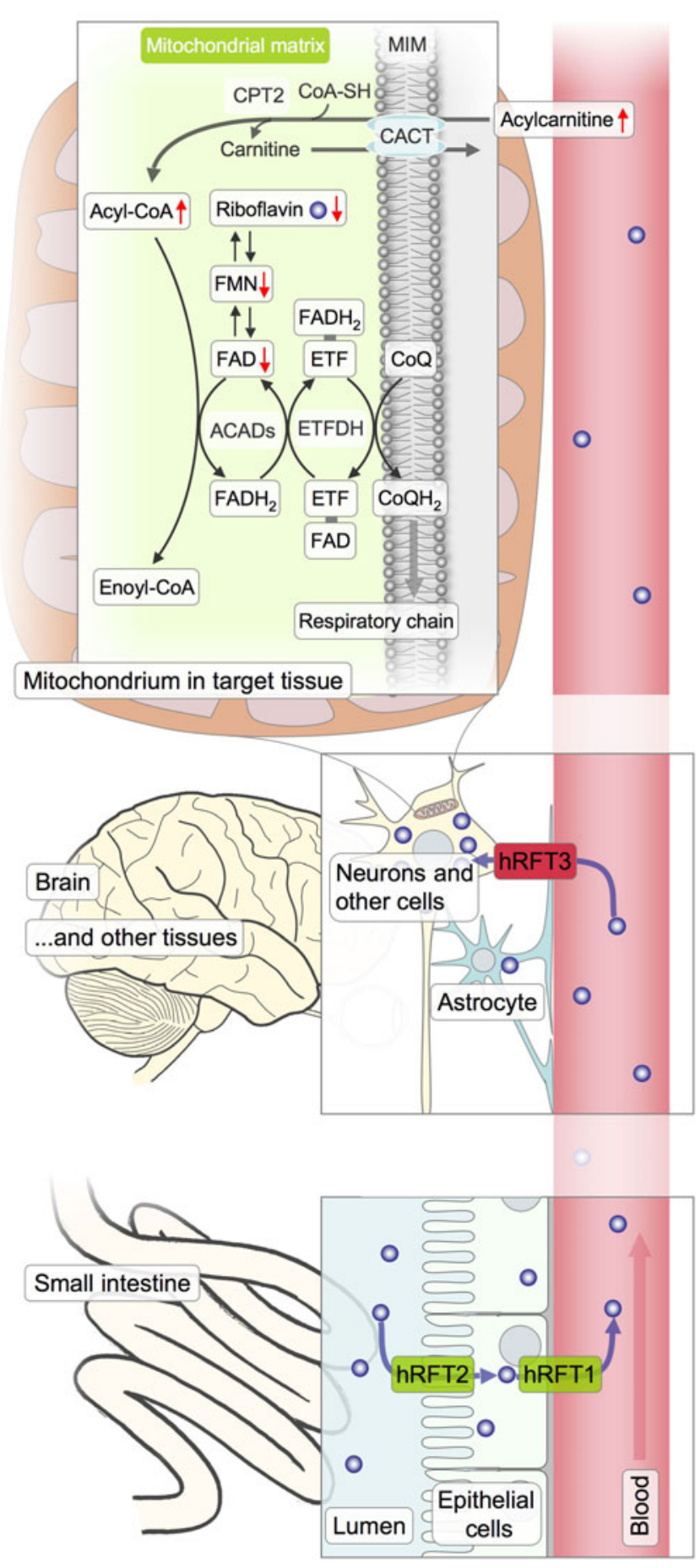

elevated acylcarnitine levels as found in patients with decreased levels of FAD argue for impaired riboflavin uptake from the blood into the cell (Fig. 3). Of note, clinically relevant disturbance of brain metabolism despite normal serum levels has also been reported for another vitamin, thiamine. Missense mutations in SLC19A3, coding for human thiamine transporter 2, cause a Wernicke encephalopathylike disease. Thiamine serum levels were normal and the 
disorder was responsive to oral high-dose thiamine treatment (Kono et al 2009).

An oral supplementation with riboflavin $(10 \mathrm{mg} / \mathrm{kg}$ body weight/day) has been initiated in the SLC52A2-mutant individual presented in this study and led to a normalization of six out of seven altered acylcarnitine species and moderate clinical improvement. Nevertheless, longer follow up of a larger cohort is necessary to judge the clinical effectiveness of riboflavin in this subtype of BVVLS. Although our in vitro studies indicate a residual function of hRFT3, one has to keep in mind that the gradient from blood to brain is likely to be significantly higher (Nagatsu et al 1967) than from intestine to epithelial cells or blood. In addition, superfluous riboflavin undergoes renal excretion thus limiting the possible increase in blood riboflavin concentration by supplementation.

In summary, we demonstrate the power of next generation sequencing in the genetic diagnosis of rare metabolic disorders providing the basis for a mechanistic therapy in some cases, like in our patient a high-dose supplementation with riboflavin. The causal nature of the mutations was confirmed by in vitro analysis of transporter activities, thereby substantiating the role of defective riboflavin transport in the pathogenesis of BVVLS. The clinical overlap with motor neuron diseases such as amyotrophic lateral sclerosis bridges the gap to more common disorders and a better understanding of riboflavin metabolism might help to pinpoint the molecular defect in these entities while providing a therapeutic perspective.

Acknowledgments We thank the affected individual and her family for their participation in the study. We acknowledge the technical support of Evelyn Botz and Carola Fischer.

Web resources The URL for data presented herein is as follows: Online Mendelian Inheritance in Man (OMIM), http://www.omim.org Exome Variant Server (EVS) of the NHLBI GO Exome Sequencing Project, Seattle, WA, http://evs.gs.washington.edu/EVS/

Funding T.M. and H.P. were supported by the Impulse and Networking Fund of the Helmholtz Association in the framework of the Helmholtz Alliance for Mental Health in an Ageing Society (HA-215) and the German Federal Ministry of Education and Research (BMBF)funded Systems Biology of Metabotypes grant (SysMBo 0315494A) and German Network for Mitochondrial Disorders (mitoNET 01GM0867). T.M. is supported by the BMBF-funded German Center for Heart Research. T.M. and T.M.S. were supported by the European Commission 7th Framework Program (N. 261123), the Genetic European Variation in Disease Consortium, and the German Ministry for Education and Research (01GR0804-4). This study was supported in part by a grant-in-aid for Scientific Research (KAKENHI) from the Ministry of Education, Science, Culture and Sports of Japan.

\section{References}

Anand G, Hasan N, Jayapal S, Huma Z, Ali T, Hull J et al (2012) Early use of high-dose riboflavin in a case of Brown-Vialetto-Van Laere syndrome. Dev Med Child Neurol 54(2):187-189

Bosch AM, Abeling NG, Ijlst L, Knoester H, van der Pol WL, Stroomer AE et al (2011) Brown-Vialetto-Van Laere and Fazio Londe syndrome is associated with a riboflavin transporter defect mimicking mild MADD: a new inborn error of metabolism with potential treatment. J Inherit Metab Dis 34(1):159-164

Brown CH (1894) Infantile amyotrophic lateral sclerosis of the family type. J Nerv Ment Dis 21:707-716

Foraker AB, Khantwal CM, Swaan PW (2003) Current perspectives on the cellular uptake and trafficking of riboflavin. Adv Drug Deliv Rev 55(11):1467-1483

Goodman SI (1981) Organic aciduria in the riboflavin-deficient rat. Am J Clin Nutr 34(11):2434-2437

Green P, Wiseman M, Crow YJ, Houlden H, Riphagen S, Lin JP et al (2010) Brown-Vialetto-Van Laere syndrome, a ponto-bulbar palsy with deafness, is caused by mutations in c20orf54. Am J Hum Genet 86(3):485-489

Ho G, Yonezawa A, Masuda S, Inui K, Sim KG, Carpenter K et al (2010) Maternal riboflavin deficiency, resulting in transient neonatal-onset glutaric aciduria Type 2, is caused by a microdeletion in the riboflavin transporter gene GPR172B. Hum Mutat 32(1):E1976-E1984

Kono S, Miyajima H, Yoshida K, Togawa A, Shirakawa K, Suzuki H (2009) Mutations in a thiamine-transporter gene and Wernicke'slike encephalopathy. N Engl J Med 360(17):1792-1794

Mayr JA, Haack TB, Graf E, Zimmermann FA, Wieland T, Haberberger B et al (2012) Lack of the mitochondrial protein acylglycerol kinase causes sengers syndrome. Am J Hum Genet 90 (2):314-320

Nagatsu T, Nagatsu-Ishibashi I, Okuda J, Yagi K (1967) Incorporation of peripherally administered riboflavine into flavine nucleotides in the brain. J Neurochem 14(2):207-210

Sathasivam S (2008) Brown-Vialetto-Van Laere syndrome. Orphanet J Rare Dis 3:9

Yao Y, Yonezawa A, Yoshimatsu H, Masuda S, Katsura T, Inui K (2010) Identification and comparative functional characterization of a new human riboflavin transporter hRFT3 expressed in the brain. J Nutr 140(7):1220-1226

Yonezawa A, Masuda S, Katsura T, Inui K (2008) Identification and functional characterization of a novel human and rat riboflavin transporter, RFT1. Am J Physiol Cell Physiol 295(3):C632-C641 